\title{
Casting Issues of Thick-Walled High Pressure Die Castings
}

\author{
D. Molnar, A. Kiss, Cs. Majoros \\ University of Miskolc, Institute of Foundry, daniel.molnar@uni-miskolc.hu
}

Abstract. Die casting is a manufacturing process for producing accurately dimensioned, sharply defined, smooth or textured surface metal parts. It is accomplished by injecting liquid metal at fast velocity and under high pressure into reusable steel dies. Compared to other casting processes, die casting is at the top end of both velocity and pressure. The high velocity translates into a very turbulent flow condition. The process is often described as the shortest distance between raw material and the finished product.

Pre-fill is a process technique that is the result of significantly delaying the start of fast shot beyond the "metal at the gate" position. It can be stated as a percentage of cavity fill or as a distance beyond metal at the gate.

\section{Process description}

In high pressure die casting the liquid melt is charged into the shot sleeve according to the chamber fill ratio. The time of metal charging affects the cycle time, productivity and the profitability. The target of charging is the air displacement from the chamber through the casting cavity, so the piston moves forward with a controlled slow velocity and the melt accumulates on the frontal face of it. During the movement of the piston waves are developing in the melt, and move along the sleeve while displaces air. The first phase is over when the melt fills the whole shot sleeve cavity which indicates a switching sign. This switching sing starts the second phase when the movement of the piston is fast and the cavity fills totally until the piston abrupt stops and hit the liquid melt. At this moment the kinetic energy transforms into pressure energy and frictional thermal energy. In the third phase the entrapped air bubbles and the shrinkage cavities are compressed with a high pressure during solidification.

\section{Pre-fill technology}

The simplified model of high pressure die casting can be seen in Fig. 1.

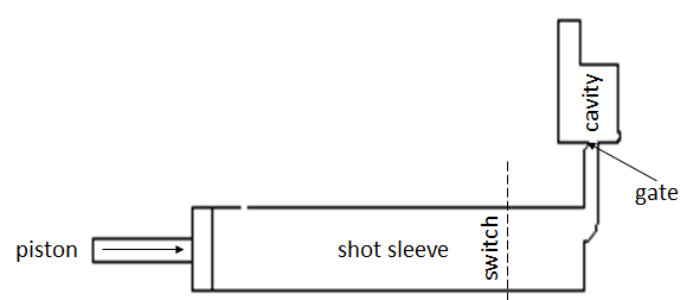

Fig.1. Simplified model of high pressure die casting 
In the common high pressure die casting process the melt never enter the cavity in the first phase, because the cross section of the gate is usually small and by the high heat conduction of the die material the melt would solidify in the gate.

In case of castings with heavy sections, the dimension of the gate is much bigger and pre-filling can be applied which means that a part of the cavity is filled during the first phase with a low piston velocity.

Pre-filling is a special matter, where the switching point is not controlled by the chamber fill ratio of the shot sleeve. The phases of the process must be redefined, and the lengths of the first and second phases and the position of the switching point must be calculated with a different method. Thus the high pressure die casting process can be divided as follows:

1st phase: pre-filling; $2^{\text {nd }}$ phase: cavity filling; $3^{\text {rd }}$ phase: solidification.

The target of pre-filling is the minimization of air entrapments in the cavity. The slow speed of the piston effects slow gate velocity with much lower kinetic energy. If the sum values of internal friction and wall friction together are higher than the kinetic energy, the forward moving melt expels the air from the gate area. If the piston velocity is too slow during pre-filling, the melt can solidify in the gate, if it is too fast, the friction forces cannot negotiate the kinetic energy and the melt splashes to the opposite wall and entraps air. [1-2]

Following are some of the characteristics that make it easier to develop a pre-fill process.

- Timing is everything: if there are multiple gate entries then it is almost essential that metal begin to enter the die cavity at the same time. The runner must be pressurized enough to prevent stalling due to gravity.

- Single cavity tools: from a process development perspective, it is desirable to have only one cavity to deal with it.

- Bottom filling gate: when applying pre-fill it is desirable to have a bottom filling casting. It reduces turbulence and expels air at the top of the cavity.

- Repeatable biscuit length: since biscuit length affects the actual metal at the gate position it is greatly beneficial to have a repeatable biscuit. Anything that changes the percentage of pre-fill will have a negative effect on the repeatability of the casting quality.

- Thicker walled castings: castings that have heavier wall sections will generally have more tolerance to a delayed fast shot. Conversely if the casting has some relatively thin features these may not form completely when attempting pre-fill.

- Volume of the casting: it seems that the larger the casting, the more likely it is to benefit from prefilling. [3]

\section{Design of experiments}

The examined casting is made of EN AC-46000 aluminium alloy, overall dimensions are $250 \times 350 \times 285$ $\mathrm{mm}$ and the average wall thickness is $4,5 \mathrm{~mm}$. Geometry of the casting and the positions of the switching points can be seen in Fig. 2. A: switching point at the gate; B: switching point at the fin; C: switchingpoint after the fin. 


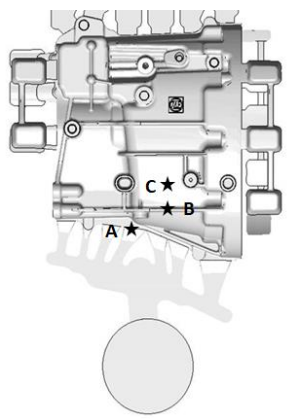

Fig. 2. Casting geometry and the positions of the switching points

The experiments can be divided as follows:

I. Determination of the technological parameters using formulas for common high pressure die casting, without taking into account the pre-filling.

II. Determination of the technological parameters with foundry tests, searching for the optimum value of parameters.

III. Determination of the technological parameters using modified formulas for pre-filling.

In the first experiments technological parameters were determined using formulas for common high pressure die casting, without taking into account the pre-filling. The calculations were carried out using the ForCasts software [4]. A test production run with these parameters and all castings were Xray tested. The scrap ratio was high, the usual defects were air entrapments and shrinkage. Because the desired second phase piston velocity was not possible to reach by the OMS-2350 casting machine and the multiplication pressure reached the maximum value, the amount of the entrapped air must be minimized.

In the foundry tests several technological parameters were examined one by one. To find the optimal value of a parameter, a design of experiment was determined which can be seen in Table 1.

\begin{tabular}{|c|c|c|c|}
\hline Experiment & Parameter & Nr. of iterations & Best value \\
\hline 1 & Temperature & 1 & $720^{\circ} \mathrm{C}$ \\
\hline 2 & Switching position & 6 & $715 \mathrm{~mm}$ \\
\hline 3 & Pressure during solidification & 5 & $490 \mathrm{bar}$ \\
\hline 4 & Time of squeeze-pin delay & 6 & $7,5 \mathrm{~s}$ \\
\hline 5 & Slow piston velocity & 5 & $0.35 \mathrm{~m} / \mathrm{s}$ \\
\hline 6 & Fast piston velocity & 3 & $3.4 \mathrm{~m} / \mathrm{s}$ \\
\hline \multicolumn{4}{|r}{ Table 1. Design of Experiment } \\
\end{tabular}

\section{Calculation of pre-filling with modified formulas}

As a result of the experiments and the test production, it is clear that the amount of the entrapped air in the casting cavity must be decreased. This decrement can be reach by using pre-fill technology. In this case the formulas for common high pressure die casting must be modified and new definitions must be determined. The inserted metal quantity in the second phase can be seen in Fig. 3. 


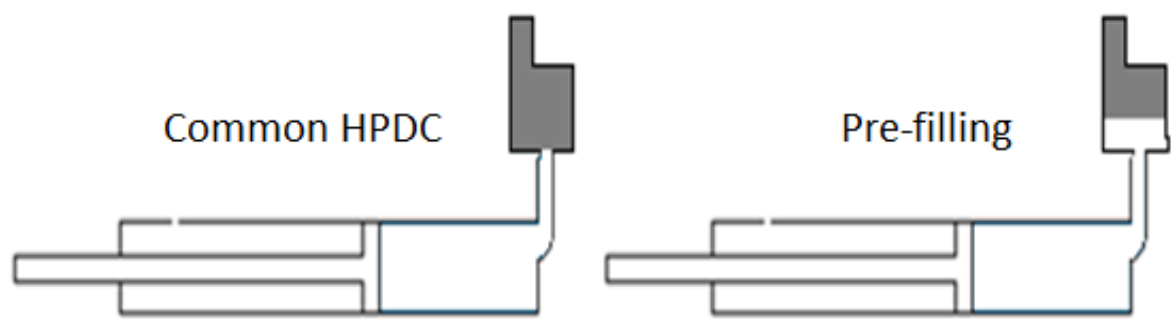

Fig. 3. Inserted metal in the second phase

The volume of pre-filled metal is the amount of metal, which is entered the cavity before the second phase. The volume of pre-filling can be calculated as follows:

\begin{tabular}{|c|ll|}
\hline \multicolumn{2}{|c|}{ Volume of pre-filling } \\
\hline Simplified equation & \multicolumn{1}{c|}{ Detailed equation (Eq.1.) } \\
\hline$V_{\text {pre }}=a \cdot A_{c}$ & \multicolumn{2}{|c|}{$V_{\text {pre }}=\left\{L_{s p}-\left[L_{c} \cdot\left(1-F_{g}\right)+\frac{V_{g s}}{A_{c}}\right]\right\} \cdot A_{c}$} \\
\hline where, & \multicolumn{1}{c|}{ where, } \\
\hline$a=L_{s p}-(b+c)$ & $\mathrm{V}_{\mathrm{pre}}:$ & volume of pre-filled metal, $\mathrm{cm}^{3}$ \\
\hline$b=L_{c} \cdot\left(1-F_{g}\right)$ & $\mathrm{L}_{\mathrm{sp}}:$ & position of the switching point, mm \\
& $\mathrm{L}_{\mathrm{c}}:$ & active length of the chamber, mm \\
& $\mathrm{F}_{\mathrm{g}}:$ & chamber fill ratio, \% \\
& $\mathrm{V}_{\mathrm{gs}}:$ & volume of the gating system, $\mathrm{m}^{3}$ \\
& $\mathrm{~A}_{\mathrm{c}}:$ & cross section of the chamber, $\mathrm{m}^{2}$ \\
\hline$A_{c}$ &
\end{tabular}

(a): Describes the piston displacement during pre-filling.

(b): Describes the determination of the switching point by the common calculation method. This is equal to the total length of the piston displacement which is needed to fill the shot sleeve.

(c): Describes the piston displacement which is needed to fill the gating system. This is actually the ratio between the volume of the gating system and the cross section of the chamber. Whereas both the volume of the gating system and the cross section of the chamber are geometrical parameters, in case of existing die these parameters cannot be modified.

In Eq. 1. the active length of the chamber is a limited variable, but the position of the switching point is free to choose although even this parameter is not considered in the common calculation method. In Table 2. the comparison of the common calculation and the pre-filling calculation can be seen.

\begin{tabular}{|c|c|c|}
\hline $\begin{array}{c}\text { Position of the } \\
\text { switching point }\end{array}$ & Common calculation & (Modified) Pre-filling calculation \\
\hline Volumetric flow & $Q=\frac{V_{\text {casting }}}{t_{\text {pouring }}}$ & $L_{s p}=\frac{V_{\text {pre }}}{A_{c}}+L_{c} \cdot\left(1-F_{g}\right)+\frac{V_{g s}}{A_{c}}$ \\
\hline $\begin{array}{c}\text { Length of the second } \\
\text { phase }\end{array}$ & $L_{2}=\frac{V_{\text {casting }}}{A_{c}}$ & $L_{\text {caver }}$ \\
\hline
\end{tabular}

Table 2. Common and pre-filling calculation

Where, $\quad t_{\text {pour: }} \quad$ pouring time, $s$

$\mathrm{V}_{\text {casting: }} \quad$ volume of the casting, $\mathrm{m}^{3}$

$\mathrm{V}_{\text {over: }} \quad$ volume of the overflows, $\mathrm{m}^{3}$ 


$$
\mathrm{V}_{\text {casting+over: }} \quad \text { volume of casting and overflows, } \mathrm{m}^{3}
$$

If the customer determines the required volume of pre-filling $\left(\mathrm{V}_{\text {pre }}\right)$ all the other parameters can be calculated based on the given equation.

\section{Comparison of the calculation methods}

The comparison of the common and the modified calculation methods can be done by using the same initial parameters where the only distinct factor is the position of the switching point. If the common calculation method is considered the position of the switching point is not taking into account, thus the results are independent from this parameter (horizontal broken-lines in the diagram).

The initial parameters of the comparison were based on an OMS-2350 casting machine: $140 \mathrm{~mm}$ piston diameter, 880 and $990 \mathrm{~mm}$ active chamber lengths. The comparison of the results using $25 \%$ pre-filling can be seen in Fig. 4.

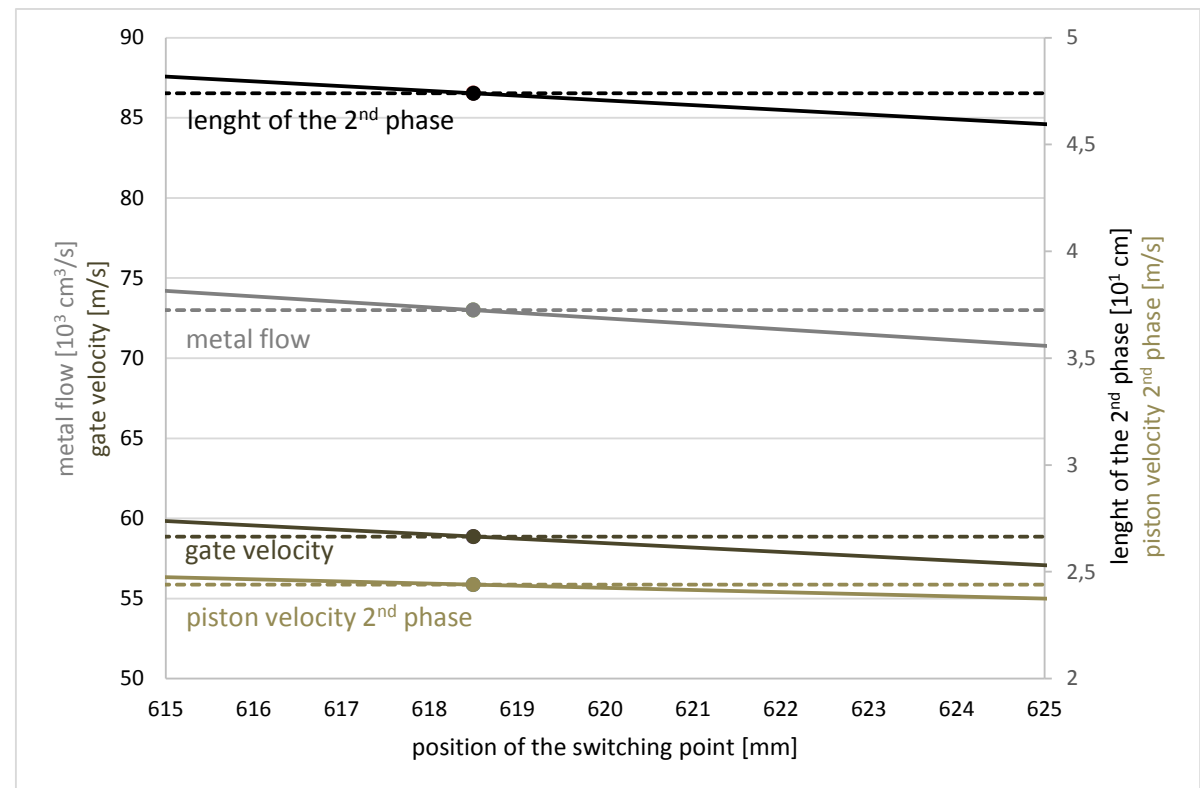

Fig. 4. Result comparison of the common- and the modified calculation methods

In Fig. 4. the results of the common calculation method are constant values (broken-lines) while the results of the modified calculation method can be described as linear lines (full lines). The results can be detailed as follows.

- If the position of the switching point is at the gate, the two calculations method gives the same value.

- If the position of the switching point is behind the gate a given part of the metal is already in the cavity before the switching sign, thus with the modified calculation all values of the parameters are lower.

If the modified calculation method is used the amount of the metal flow and the gate velocity is lower, which means lower abrasive wear of the die. If the determined piston velocity is lower, the piston impact on the liquid melt is smaller. On the other hand if the velocity of the metal flow is lower the surface quality of the casting can be worse. 


\section{Validation via simulation}

The modified calculation method was validated using the commercial NovaFlow\&Solid CV simulation software. [5-6] In the simulation experiments the following initial parameters were defined:

- Alloy:

- Pouring temperature:

- Material of the die:

- Temperature of the die:

- 1st phase velocity:

- 2nd phase velocity:

- Value of pre-filling:
EN AC-46000

$720^{\circ} \mathrm{C}$

H13 steel (orvar supreme)

$180^{\circ} \mathrm{C}$

$0.35 \mathrm{~m} / \mathrm{s}$

$3.4 \mathrm{~m} / \mathrm{s}$

$25 \%$

The comparison of the calculated and the simulated values can be seen in Fig. 5.

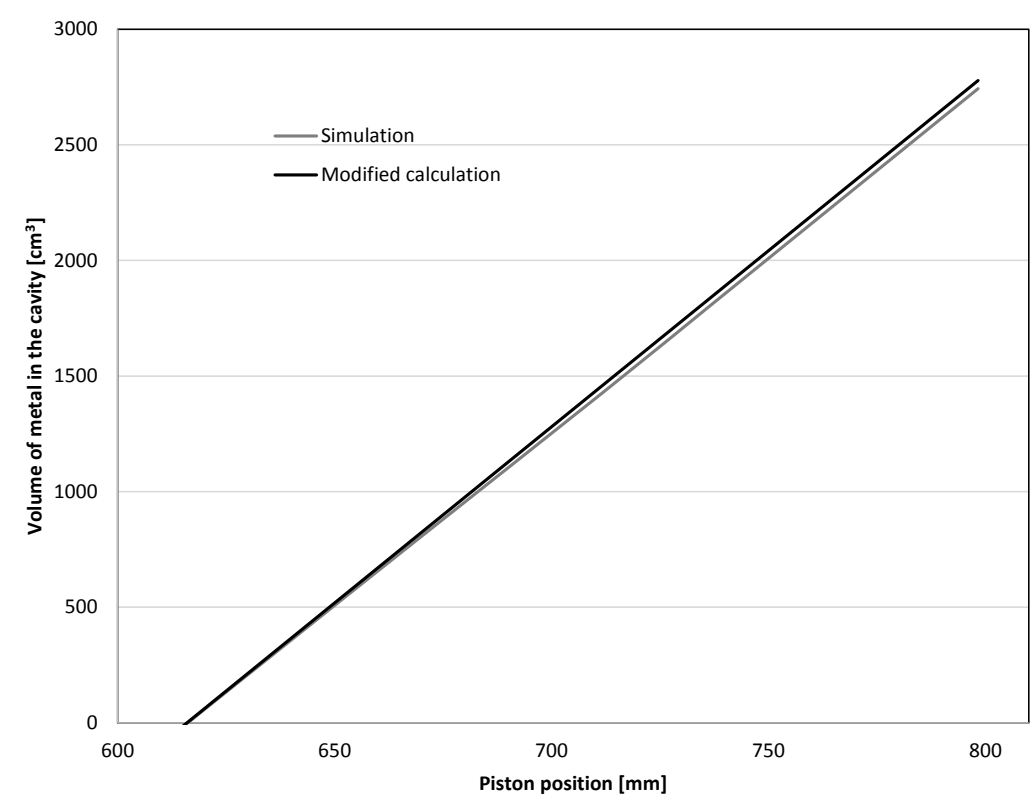

Fig. 5. The comparison of the calculated and the simulated values

The correlation coefficient between the calculated and the simulated values is: $\mathrm{R}^{2}=0.9998$. The two curves only differ to each other $\left(\Delta_{\max }: 1,4 \%\right)$ after the switching point, because in the modified calculation the volumetric contraction of the melt is not observed. Before the switching point the melt only contacts the die with a small surface, thus the calculated volume of metal in the cavity is equal. After the switching point the contact surface between the melt and the die becomes higher, the melt shrink (the volume is smaller) and this volumetric contraction is not calculated in the modified calculation method.

In the simulation experiments three different pre-filling positions were observed (A,B,C see Fig. 2.). The effects of the switching point position were examined on the:

- filling time,

- solid phase ration at the end of the filling,

- amount of the entrapped air in the gating system. 


\section{Summary}

The liquid phase ratio at the end of the filling of each pre-filling variations can be seen in Fig. 6 . The scale is the liquid phase ratio: $85-99 \%$.

In Fig. 6. the light colour represents the liquid phase and the dark colour represents the solid phase. Filling time $\left(\mathrm{t}_{\text {fill }}, \mathrm{s}\right)$ and the solid phase ratio (\%) are highlighted under the figure.

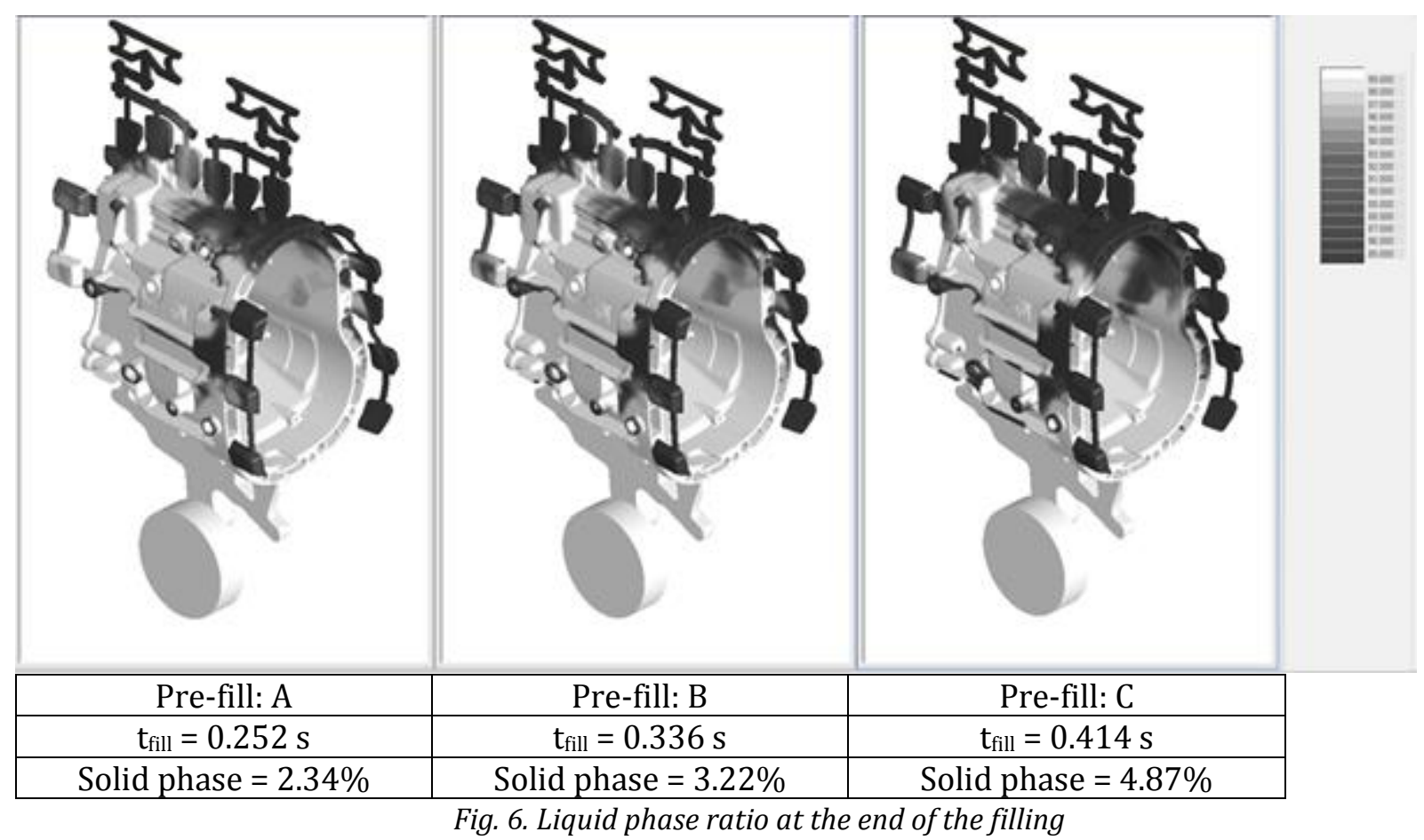

In Fig. 7. the amount of the entrapped air (black) can be seen in the 2D cut of the gating system. The definition of the 2D cut can be seen on the left side.

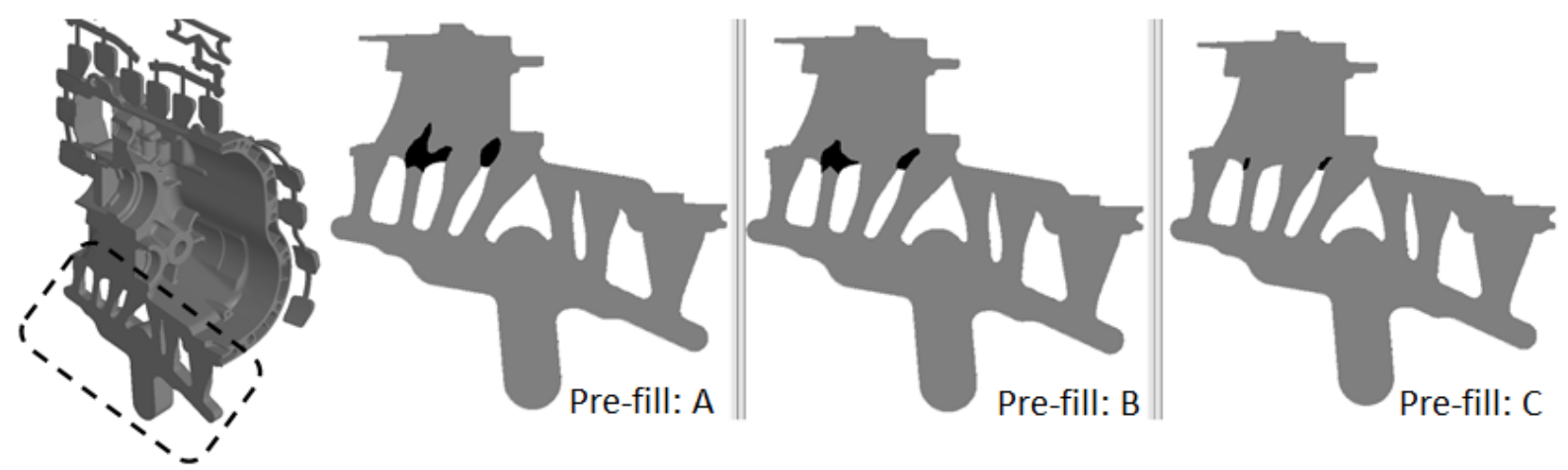

Fig. 7. Entrapped air in the gating system, 2D cut

In Fig. 6. one can see that if the switching occurs later the amount of the solid phase at the end of the filling is higher. The more metal inserted with a low piston velocity (1st phase) the filling time is longer. Observing each simulation the following statements can be made. 
- If the switching point is at the gate (pre-fill A) no pre-filling occurs, the piston accelerates to the 2nd phase velocity while the melt enters the gate. Cavity filling is fast thus the melt contacts the die surface only for a short time and it doesn't lost much heat, solidification occurs only at the filling front. In this case the amount of the entrapped air in the area of the gating system is the highest.

- If the switching point is after the gate (pre-fill B) a part of the cavity is filled with a low piston velocity. The melt contacts the die surface for a longer time, lost much more heat, thus the amount of the solidified phase during filling is higher but not as much that it would endanger the quality of the casting part. The amount of the entrapped air in the area of the gating system is lower.

- If the switching point is far after the gate (pre-fill C) a considerable part of the cavity is filled with a low piston velocity. The melt stay in the cavity for a longer time, lost a bigger amount of heat and the quantity of the solidified ratio is much higher.

- The amount of the entrapped air is low, compared to pre-fill A and B. This is because during prefilling the melt moves with a low velocity, and it doesn't have as big kinetic energy. Because of this and because of the wall friction of the die, the melt stay in the area of the gate and displaces the air which leaves the cavity through the venting system.

In case of castings with heavy sections, the dimensions of the gate is much bigger, thus pre-filling can be applied which means that a part of the cavity is filled during the first phase with a low piston velocity. The low speed of the piston effects low gate velocity with much lower kinetic energy and this results the minimization of air entrapments.

A modified calculation method is worked out to be able to calculate correct technological parameters when using pre-filling. The comparison of the common calculation method and the modified calculation method was implemented and the modified calculation was validated with simulation.

The described article was carried out as part of the EFOP-3.6.1-16-2016-00011 "Younger and Renewing University - Innovative Knowledge City - institutional development of the University of Miskolc aiming at intelligent specialisation" project implemented in the framework of the Szechenyi 2020 program. The realization of this project is supported by the European Union, co-financed by the European Social Fund.

\section{References}

[1] Matti Sirvio et al., Complete Simulation of High Pressure Die Casting Process, VTT Industrial Systems, Finland, 2015

[2] Bill Andresen, Die Casting Engineering, Marcel Dekker, New York, 2005

[3] Robert P. McClintic, Pre-Fill a Hot Topic, Die Casting Engineer, 2015/1

[4] Forcasts Software Manual, Arbeitsgemeinschaft Metallguss GmbH, Aalen, 2012

[5] Jesper Hattel, Fundamentals of Numerical Modelling of Casting Processes, Polyteknisk, Lyngby, 2005

[6] NovaFlow\&Solid User Guide, Novacast Systems AB, Ronneby, 2016 